\title{
High efficiency phosphorescent organic light-emitting diodes using carbazole-type triplet exciton blocking layer
}

\author{
Sung Hyun Kim and Jyongsik Jang \\ School of Chemical and Biological Engineering, Seoul National University, Shinlim-dong, Kwanak-gu, \\ Seoul 151-742, Korea \\ Jun Yeob Lee La $^{2}$ \\ Department of Polymer Science and Engineering, Dankook University, Hannam-dong, Yongsan-gu, \\ Seoul 140-714, Korea
}

(Received 20 March 2007; accepted 2 May 2007; published online 31 May 2007)

\begin{abstract}
Device performances of green phosphorescent organic light-emitting diodes using $\left(4,4^{\prime}-N, N^{\prime}\right.$-dicarbazole)biphenyl (CBP) and $N, N^{\prime}$-dicarbazolyl-3,5-benzene (mCP) as an exciton blocking layer were investigated. $\mathrm{CBP}$ and $\mathrm{mCP}$ were introduced between hole transport layer and emitting layer to block triplet exciton quenching and efficient hole transport to emitting layer. The efficiency of green devices could be improved by more than three times by using $\mathrm{mCP}$ exciton blocking layer. (C) 2007 American Institute of Physics. [DOI: 10.1063/1.2742788]
\end{abstract}

Phosphorescent organic light-emitting diodes (PHOLEDs) are promising as light-emitting materials in OLEDs due to their merit of high efficiency. $100 \%$ internal quantum efficiency could be realized in green PHOLEDs Ref. 1 and high power efficiency of $77 \mathrm{~lm} / \mathrm{W}$ was reported in PHOLEDs. 2

There have been many studies about light emission and high efficiency in PHOLEDs. ${ }^{3-7}$ Many different device architectures were tried to improve the light-emitting efficiency of PHOLEDs. A hole blocking layer or exciton blocking layer (EBL) was introduced in PHOLEDs to block hole injection from light-emitting layer (EML) to electron transport layer and it was effective to get high efficiency in PHOLEDs. ${ }^{3-5}$ An electron blocking layer was also used in blue PHOLEDs to block electron injection from EML to hole transport layer (HTL). ${ }^{5} \quad$ Fac-tris(1-phenylpyrazolato- $\left.N, C 2^{\prime}\right) \quad$ iridium $\left[\operatorname{Ir}(\mathrm{ppz})_{3}\right]$ with its lowest unoccupied molecular orbital (LUMO) level of $1.7 \mathrm{eV}$ was efficient as an electron blocking material. Other than these, a double EML structure was used and high quantum efficiency of $19.3 \%$ was reported. ${ }^{2,6}$ A graded doping structure was studied by our group and it also gave a long lifetime as well as high power efficiency. ${ }^{7}$

In this work, we studied the use of $\left(4,4^{\prime}-N\right.$, $N^{\prime}$-dicarbazole)biphenyl (CBP) and $N, N^{\prime}$-dicarbazolyl3,5-benzene $(\mathrm{mCP})$ as an EBL to get high efficiency in green PHOLEDs. A detailed mechanism for exciton blocking of $\mathrm{CBP}$ and $\mathrm{mCP}$ was clarified and device performances were investigated.

The standard device structure used in this experiment was indium tin oxide $(150 \mathrm{~nm}) / N, N^{\prime}$-diphenyl- $N, N^{\prime}$-bis[4-(phenyl- $m$-tolyl-amino)-phenyl]-biphenyl-4, $\quad 4^{\prime}$-diamine $(60 \mathrm{~nm}) / N, \quad N^{\prime}$-di(1-naphthyl)- $N, \quad N^{\prime}$-diphenylbenzidine (NPB) $\quad(30 \mathrm{~nm}) / \mathrm{PH} 1: \quad$ tris $(2$-phenylpyridine) iridium $\left[\operatorname{Ir}(\text { ppy })_{3}\right]$ (30 nm, 5\% doping)/biphenoxy-bi(8-hydroxy-3methylquinoline) aluminum $(5 \mathrm{~nm}) /$ tris $(8$-hydroxyquinoline $)$ aluminum $(20 \mathrm{~nm}) / \mathrm{LiF}(1 \mathrm{~nm}) / \mathrm{Al}(200 \mathrm{~nm})$. Three devices with different HTL structures were fabricated to investigate the effect of CBP and mCP EBLs on device performances.

${ }^{a}$ Author to whom correspondence should be addressed; FAX: 82-2-7092614; electronic mail: leej17@dankook.ac.kr
Device I had NPB (30 nm) as a HTL and device II had both NPB $(20 \mathrm{~nm})$ and CBP $(10 \mathrm{~nm})$ as a double layer HTL, while device III had NPB $(20 \mathrm{~nm})$ and $\mathrm{mCP}(10 \mathrm{~nm})$ as a double layer HTL. PH1 was supplied from Merck Co. and it has a spirobifluorene-type backbone structure with high electron transport properties because of spirobifluorene units. The triplet band gap of PH1 was $2.4 \mathrm{eV}$ and the highest occupied molecular orbital (HOMO) and LUMO were 5.9 and $2.8 \mathrm{eV}$, respectively. The current density-voltageluminance characteristics of the devices were measured with Keithley 2400 source measurement unit and PR 650 spectrophotometer.

It is important to confine excitons in EML to increase the recombination efficiency of holes and electrons in OLEDs by blocking charge carriers and exciton diffusion out of EML. ${ }^{3}$ Hole and exciton blocking in PHOLED was effective in electron transport layer side and electron and exciton blocking in HTL side is expected to give high recombination efficiency through charge and exciton confinement inside EML. Exciton blocking materials should have good hole transport properties to transport holes from hole injection layer to EML, and it is required to have a wide triplet band gap to block exciton diffusion and quenching. ${ }^{8}$ In addition, it should have electron blocking function to prevent electron overflow to HTL. Therefore, CBP and $\mathrm{mCP}$ were chosen as an exciton blocking material in this work and their role as an EBL was investigated.

Figure 1 shows current density-voltage-luminance characteristics of green devices with $\mathrm{CBP}$ and $\mathrm{mCP}$ EBL compared with standard devices. The current densities of device I with NPB as a HTL and device II with NPB and CBP double layer as a HTL were similar, while device III with $\mathrm{NPB}$ and $\mathrm{mCP}$ as a HTL was rather low compared with that of devices I and II. The similar current density in devices I and II is due to similar energy barrier between organic layers. Energy level diagrams of devices II and III are schematically described as an inset in Fig. 1. The energy barrier for hole injection from NPB to CBP is $0.4 \mathrm{eV}$ and there is no energy barrier for hole injection from CBP to PH1. Device I also has $0.4 \mathrm{eV}$ HOMO difference between NPB and PH1, and the hole injection barrier between HTL and EML in device I is 


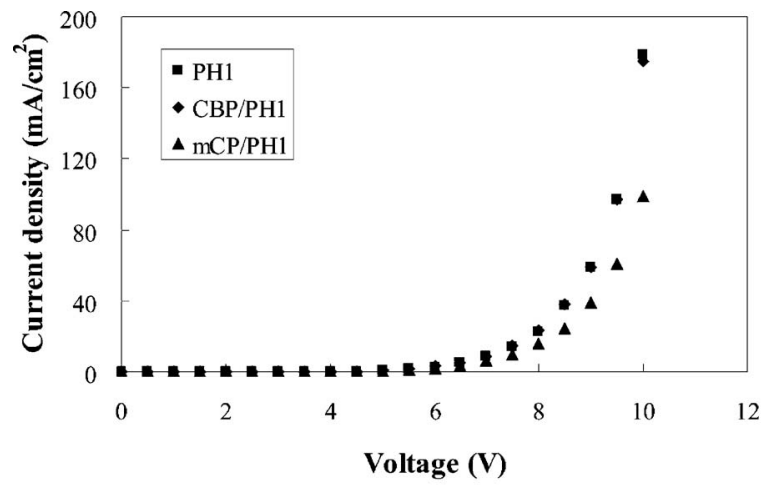

(a)

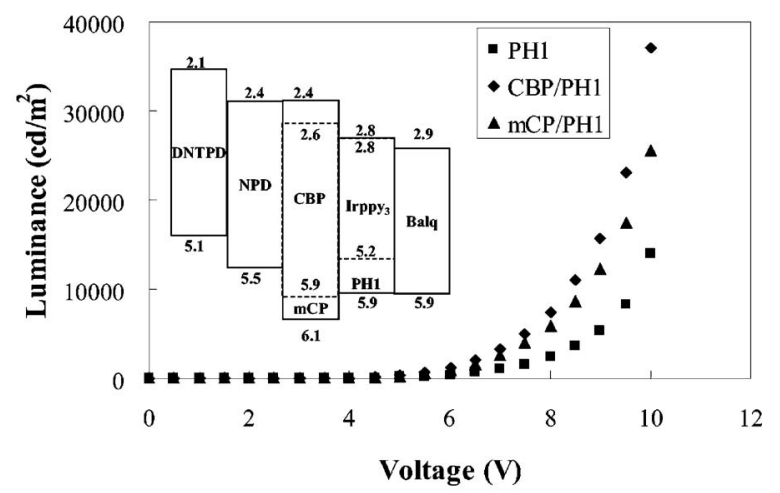

(b)

FIG. 1. Current density-voltage curves of green phosphorescent devices with different HTL structures (energy level diagram as an inset): (a) currentvoltage and (b) luminance-voltage.

the same as that of device II. Therefore, hole injection in devices I and II can be similar, leading to similar current density in the device. Compared with device II, device III has a large energy barrier of $0.6 \mathrm{eV}$ between NPB and $\mathrm{mCP}$, resulting in low current density even though there is no energy barrier between $\mathrm{mCP}$ and PH1. Luminance-voltage characteristics of three devices are also shown in Fig. 1. The luminance of device II with CBP EBL was higher than that of other devices, and device III showed the lowest luminance value at the same driving voltage. Considering similar current densities of devices I and II, the high luminance of device II compared with device I indicates that exciton formation and light emission are efficient in device II.

Quantum efficiency-luminance of three devices was plotted in Fig. 2 based on current density and luminance of devices. Device III with $\mathrm{mCP}$ as an EBL showed the best quantum efficiency value of $14 \%$ and device II with CBP EBL also exhibited high efficiency of $11.9 \%$ compared with $3.4 \%$ of device I at $1000 \mathrm{~cd} / \mathrm{m}^{2}$. As expected from energy level diagram in Fig. 1, mCP can play a role of an electron blocking layer as well as an EBL. Electrons can be blocked by $\mathrm{mCP}$ and triplet exciton quenching can be reduced by $\mathrm{mCP}$ because of the wide triplet band gap of $\mathrm{mCP}(2.9 \mathrm{eV})$, resulting in an efficient recombination of holes and electrons inside the emitting layer. Even though NPB is efficient as an electron blocking layer due to the high LUMO energy barrier of $0.4 \mathrm{eV}$ between NPB and PH1, the triplet energy level of NPB is only $2.3 \mathrm{eV},{ }^{5}$ which is not high enough for exciton blocking from EML because the triplet energy level of host and dopant materials is $2.4 \mathrm{eV}$. Compared with NPB, mCP has a triplet energy level of $2.9 \mathrm{eV}$ (Ref. 5) and it can block Downloaded 23 Nov 2007 to 147.46.244.154. Redistribution subject

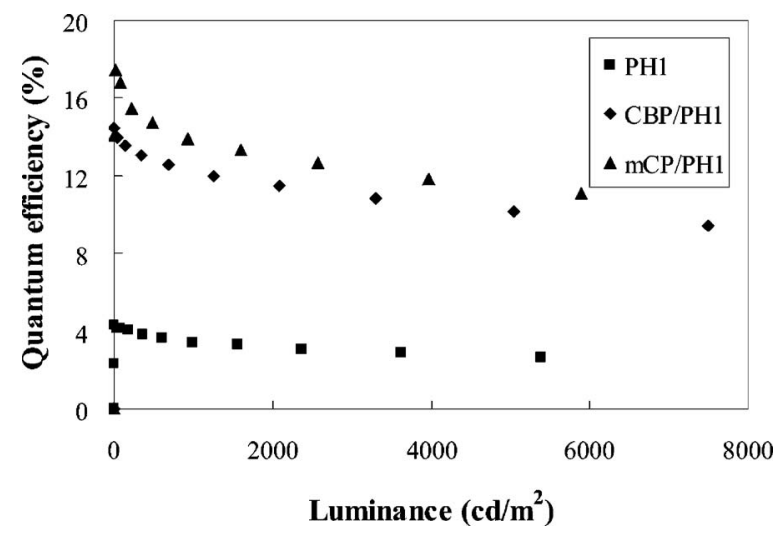

FIG. 2. Quantum efficiency-luminance curves of green phosphorescent devices with different HTL structures.

triplet exciton quenching. CBP can also act as an EBL due to a wide triplet band gap of $2.6 \mathrm{eV}$ even though it is not so efficient as $\mathrm{mCP}$ as an electron blocking layer. The exciton blocking function of $\mathrm{mCP}$ was confirmed from photoluminescence (PL) spectra. Figure 3 shows PL spectra of standard device and device with carbazole-type EBL. PL intensity was high in the device with CBP and mCP EBL, indirectly proving that $\mathrm{CBP}$ and $\mathrm{mCP}$ reduce triplet exciton quenching by NPB. Even though $\mathrm{mCP}$ has a wider triplet band gap than CBP, PL intensity was quite similar to each other. This result indicates that $\mathrm{mCP}$ and $\mathrm{CBP}$ show similar performances as a triplet EBL. Considering similar exciton blocking effect but different efficiency in CBP and $\mathrm{mCP}$ devices, the high efficiency in $\mathrm{mCP}$ device might be due to good hole-electron balance in emitting layer which originated from less hole flow in $\mathrm{mCP}$ device.

Figure 4 shows electroluminescence spectra of three devices. Green emission with peak position of $515 \mathrm{~nm}$ was observed in all devices which originated from $\operatorname{Ir}(\mathrm{ppy})_{3}$ triplet emission. In addition to green emission, device I also showed another broad peak at $458 \mathrm{~nm}$ which is assigned to NPB emission, ${ }^{4}$ while devices II and III exhibited no emission in blue wavelength range. The NPB emission in device I may be due to hole accumulation at the interface between NPB and PH1 and electron overflow from PH1 to NPB. In general, holes are strongly trapped by dopant materials in $\operatorname{Ir}(\mathrm{ppy})_{3}$ doped devices ${ }^{7}$ and holes can be accumulated between NPB and PH1 because hole transport through $\operatorname{Ir}(\mathrm{ppy})_{3}$ is limited. The accumulated holes can recombine with some electrons injected from EML inside NPB, giving rise to NPB

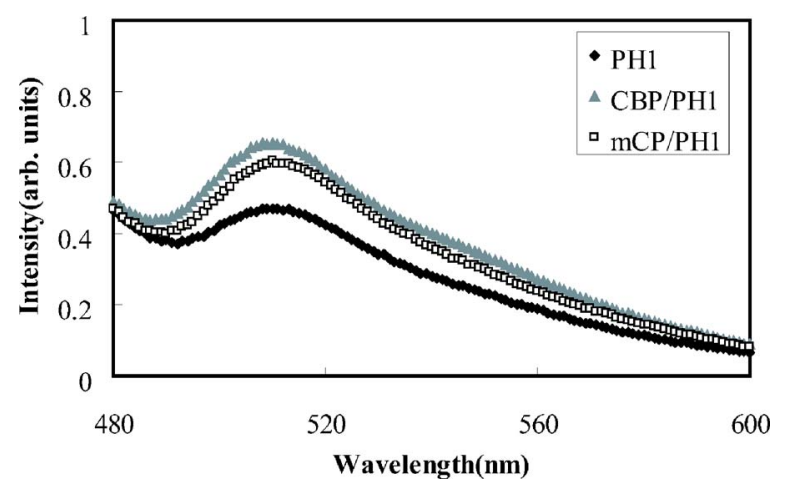

FIG. 3. Photoluminescence spectra of green phosphorescent devices with

different hole transport layers. 


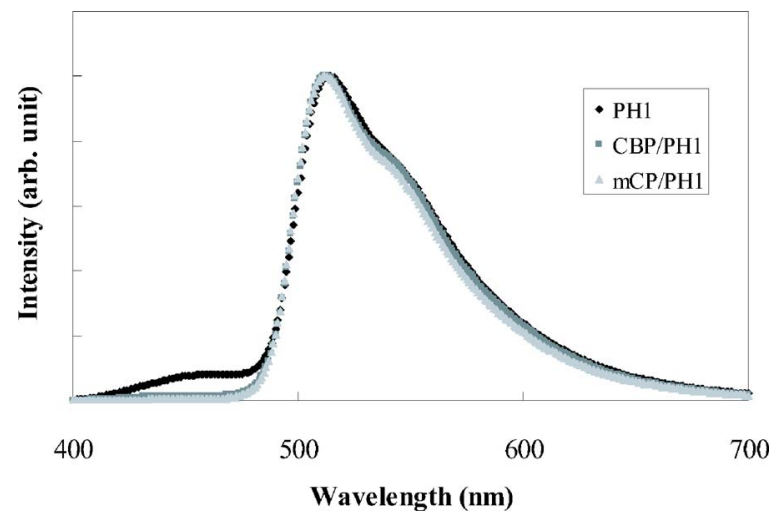

FIG. 4. Electroluminescence spectra of green phosphorescent devices with different hole transport layers.

emission. ${ }^{6}$ Compared with device I with blue emission, no blue emission in devices II and III is due to the reduced hole accumulation at the interface between HTL and EML. Holes are injected from $\mathrm{CBP}$ or $\mathrm{mCP}$ layer and can be transported through PH1 host materials. Therefore, hole accumulation can be greatly reduced and most of the holes can recombine with electrons in EML, leading to efficient green emission. No blue emission in PH1 device with CBP EBL supports this explanation. The LUMO level of CBP is only $2.6 \mathrm{eV}$ and electrons can be efficiently injected from PH1 to the CBP and NPB layer, which will induce NPB blue emission through recombination with accumulated holes at the interface. However, NPB emission was not observed in the CBP device, which supports the fact that efficient hole transport dominates light emission in $\mathrm{PH} 1$ device with $\mathrm{CBP}$ or $\mathrm{mCP}$ EBL. It can be inferred from electroluminescence spectra that the recombination zone of holes and electrons was shifted from HTL side to EML by EBL, considering the disappearance of blue emission in devices II and III.

In summary, the use of $\mathrm{CBP}$ and $\mathrm{mCP}$ as an excition blocking layer in green PHOLEDs was effective to get high luminance efficiency, and quantum efficiency was enhanced from $3.4 \%$ to $14.0 \%$ by $\mathrm{mCP}$ EBL. Both CBP and $\mathrm{mCP}$ were efficient as an EBL, and there was no emission from NPB in the device with CBP and mCP EBL.

${ }^{1}$ C. Adachi, M. Baldo, M. E. Thompson, and S. R. Forrest, J. Appl. Phys. 90, 5048 (2001).

${ }^{2}$ G. He, M. Pfeiffer, K. Leo, M. Hofmann, J. Birnstock, R. Pudzich, and J. Salbeck, Appl. Phys. Lett. 85, 3911 (2004).

${ }^{3}$ M. A. Baldo, M. E. Thompson, and S. R. Forrest, Pure Appl. Chem. 71, 2095 (1999).

${ }^{4}$ M. Ikai, S. Tokito, Y. Sakamoto, T. Suzuki, and Y. Taga, Appl. Phys. Lett. 79, 156 (2001).

${ }^{5}$ V. Adamovich, S. R. Cordero, P. I. Djurovich, A. Tamayo, M. E. Thompson, B. Andrade, and S. R. Forrest, Org. Electron. 4, 77 (2003).

${ }^{6}$ X. Zhou, D. S. Qin, M. Pfeiffer, J. Blochwitz, A. Werner, J. Crechsel, B. Maennig, K. Leo, M. Bold, P. Erk, and H. Hartmann, Appl. Phys. Lett. 81, 4070 (2002).

${ }^{7}$ J. Y. Lee, Appl. Phys. Lett. 89, 153503 (2006).

${ }^{8}$ Y. Wang, Appl. Phys. Lett. 85, 4848 (2004). 